\title{
一种新型 1,3,4-噁二嗪酮化合物的合成及其晶体结构
}

\author{
孙宏顺 ${ }^{a, b}$ 王建强 $^{a}$ 谭文文 $^{b}$ 杨海东 ${ }^{b}$ \\ 李玉龙 ${ }^{b}$ 沈临江*,a 郭 成 ${ }^{a}$ \\ ( ${ }^{a}$ 南京工业大学化学与分子工程学院 南京 211816) \\ ( $b$ 南京科技职业学院化学工程系 南京 210048)
}

摘要 以苯并噻吩-2-甲酸为原料, 经酯化、肼解、肼的酰基化和环化四步反应合成了一种新型化合物 2-(2-苯并噻吩 基)-4H-1,3,4-惡二嗪-5(6H)-酮(4). 产物结构经 ${ }^{1} \mathrm{H}$ NMR, ${ }^{13} \mathrm{C}$ NMR, ESI-MS, FT-IR, 元素分析及 X 单晶衍射表征.

关键词 苯并噻吩; 噁二嗪; 合成; 晶体结构

\section{Synthesis and Crystal Structure of a Novel 1,3,4-Oxadiazin-one Compound}

\author{
Sun, Hongshun ${ }^{a, b} \quad$ Wang, Jianqiang ${ }^{a} \quad$ Tan, Wenwen $^{b} \quad$ Yang, Haidong ${ }^{b}$ \\ Li, Yulong $^{b} \quad$ Shen, Linjiang ${ }^{*, a} \quad$ Guo, Cheng ${ }^{a}$ \\ ( ${ }^{a}$ College of Chemistry and Molecular Engineering, Nanjing Tech University, Nanjing 211816) \\ ( ${ }^{b}$ Department of Chemical Engineering, Nanjing Polytechnic Institute, Nanjing 210048)
}

\begin{abstract}
Using benzothiophene-2-carboxylic acid as a raw material, 2-(1-benzothiophen-2-yl)-4H-1,3,4-oxadiazin$5(6 H)$-one (4) was synthesized by esterification and hydrazinolysis, followed by the acylation and cyclization reactions. The novel compound 4 was characterized by ${ }^{1} \mathrm{H}$ NMR, ${ }^{13} \mathrm{C}$ NMR, ESI-MS, FT-IR, elemental analysis and X-ray single crystal diffraction.
\end{abstract}

Keywords benzothiophen; oxadiazin; synthesis; crystal structure

在新型农药研制领域, 含噁二嗪(oxadiazine)结构的 杂环化合物一直是研究热点之一 ${ }^{[1 \sim 4]}$. 尤其是以杜邦公 司开发的第一个商业化的钠通道阻断型杀虫剂狮虫威 (indoxacarb, A) 为代表 ${ }^{[5]}$, 人们合成了多种含噁二嗪结 构的新型化合物并对其性能进行了研究 ${ }^{[6 \sim 10]}$. 袁莉萍课 题组 ${ }^{[11]}$ 合成了系列 2-取代-1,3,4-惡二嗪酮类化合物 $\mathbf{B}$ (图 1), 结果发现此类化合物具有良好的除草活性, 特 别是对马唐(Digitaria sanguinalis)和苋菜(Ambrosia tricolor Linn)有较高活性.

噻吩及其衍生物是一类非常重要的有机合成中间体, 在合成杀虫剂、杀菌剂、除草剂及医药产品等方面有着 重要的作用 ${ }^{[12 ~ 15]}$. 如已开发成功的 4 个杀菌剂噻菌嗪 (thicyofen)、噻唑菌胺(ethaboxam)、硅噻菌胺(silthiopham)<smiles></smiles>

A<smiles>[R]C1=NN([R])C(=O)CO1</smiles><smiles>[R]Cc1coc(-c2cncc([Y])c2)c1</smiles>

图 1 部分含噁二嗪结构的化合物

Figure 1 Some compounds containing oxadiazine structure

*E-mail: ljshen@njut.edu.cn

Received September 25, 2015; revised October 27, 2015; published online November 6, 2015.

Project supported by the National Natural Science Foundation of China (No. 21106069) and the Key Program of Nanjing Polytechnic Institute (No. NHKY-2015-1).

国家自然科学青年基金(No. 21106069)、南京科技职业学院院级重点(No. NHKY-2015-1)资助项目. 
和吡噻菌胺均是以噻吩环为主要结构 ${ }^{[16]}$.

鉴于含噁二嗪和噻吩结构的化合物在新型农药研 制领域的广泛应用，本工作设计合成了一种 2-苯并噻 吩-1,3,4-啞二嗪酮类化合物, 并对其晶体结构进行了解 析，其合成路线如 Scheme 1 所示.

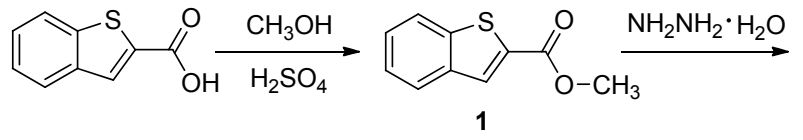

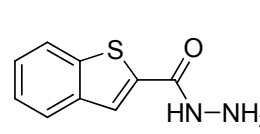

2

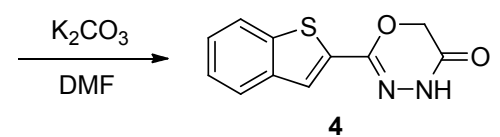

图式 1 化合物 4 的合成路线

Scheme 1 Synthesis route of compound 4

\section{1 结果与讨论}

\section{1 反应条件}

目标化合物的合成分为四步: 酯化、肼解、肼的酰 基化和环化. 第一步是苯并噻吩-2-甲酸与甲醇化反应 合成苯并噻吩-2-甲酸甲酯; 第二步是苯并噻吩-2-甲酸 甲酯与肼反应合成苯并噻吩-2-甲酰肼; 第三步是将苯 并噻吩-2-甲酰肼与氯乙酰氯反应, 在酰肼位置上引入 氯乙酰基; 最后一步为分子内成环反应，化合物 3 以 $\mathrm{K}_{2} \mathrm{CO}_{3}$ 为碱性试剂, 回流条件下发生分子内成环反应得 到 2-苯并噻吩-1,3,4-噁二嗪酮化合物 4.

2004 年, Tietze 等 ${ }^{[17]}$ 用三甲基氯硅烷为催化剂, 将 苯并噻吩-2-甲酸与甲醇回流反应 $24 \mathrm{~h}$, 得到苯并噻吩2-甲酸甲酯，收率达到 $98 \%$. 但是该反应使用的催化剂 为原料的两倍, 反应时间也较长. 因此我们仍采用硫酸 作催化剂的通用酯化方法，反应时间 $4 \mathrm{~h}$ ，产率 93\%.

2014 年, Leonczak 等 ${ }^{[18]}$ 将苯并噻吩-2-甲酸乙酯与 水合肼在乙醇中回流反应得到苯并噻吩-2-甲酰肼, 收 率 $90 \%$. 我们改用苯并噻吩-2-甲酸甲酯与水合肼在甲 醇中回流反应得到苯并噻吩-2-甲酰肼，收率达到 $92 \%$.

2004 年, Shiota 等 ${ }^{[19]}$ 报道了化合物 3 的合成，其方 法是将化合物 2 溶于乙腈中, 滴加氯乙酰氯后于室温下 反应 $16 \mathrm{~h}$, 收率 $54 \%$. 我们将溶剂改为 DMF 后，反应时 间缩短到 $4 \mathrm{~h}$, 收率提高到 $80 \%$.

有关 1,3,4-腎二嗪酮类化合物的合成方法首次见于 1929 年的文献报道(Eq. 1) ${ }^{[20]}$, 后续研究中涉及到的主要
碱化试剂有碳酸钠、碳酸氢钠、氢氧化钠、碳酸钾、碘 化钾等. 本实验中, 分别选择碳酸钠、碳酸氢钠、氢氧 化钠和碳酸钾进行了对比实验，发现以碳酸氢钠和氢氧 化钠作碱化试剂时，反应收率很低，分别为 $15 \%$ 和 $21 \%$; 以碳酸钠和碳酸钾为碱化试剂时，收率分别达到 33\%和 $48 \%$. 原因在于碳酸氢钠碱性太弱，不利于氮负离子的 生成, 氢氧化钠碱性太强会产生更多副反应, 碳酸钠和 碳酸钾碱性适中，有利于反应进行，但碳酸钾在溶剂 DMF 中的溶解度大于碳酸钠, 碱性也稍强, 更有利于反 应的进行.

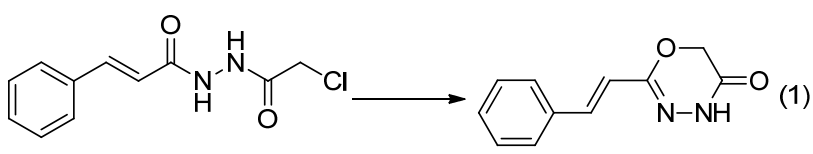

\section{2 目标化合物的波谱特征}

4 的 ${ }^{1} \mathrm{H}$ NMR 分析表明, N-H 中氢的吸收峰位于 $\delta$ 11.12 单峰处, $\delta 4.83$ 处的尖锐单峰为噁二嗪环 $\mathrm{CH}_{2}$ 中氢 的吸收峰. 在 IR 谱图中, $1686 \mathrm{~cm}^{-1}$ 处的强吸收峰为噁 二嗪酮环中 $\mathrm{C}=\mathrm{O}$ 基团的吸收峰; $3242 \mathrm{~cm}^{-1}$ 处的强吸收 峰为 $\mathrm{N}-\mathrm{H}$ 伸缩振动峰; $1398 \mathrm{~cm}^{-1}$ 处的吸收峰为 $\mathrm{C}-\mathrm{N}$ 伸缩振动峰; 1057 1182 $\mathrm{cm}^{-1}$ 处的吸收峰为噁二嗪环 中 $\mathrm{C}-\mathrm{O}-\mathrm{C}$ 醚键的伸缩振动峰; $3047 \sim 3125 \mathrm{~cm}^{-1}$ 处的 吸收峰为苯环的 $\mathrm{C}$ - $\mathrm{H}$ 伸缩振动吸收峰; 720 $864 \mathrm{~cm}^{-1}$ 处的吸收峰为苯环的 $\mathrm{C}-\mathrm{H}$ 面外弯曲振动吸收峰; $1457 \sim 1560 \mathrm{~cm}^{-1}$ 处的吸收峰为苯环的骨架振动吸收峰; $2898 \mathrm{~cm}^{-1}$ 处的吸收峰为噁二嗪环 $\mathrm{CH}_{2}$ 中的 $\mathrm{C}-\mathrm{H}$ 伸缩 振动吸收峰. 在 ${ }^{13} \mathrm{C} \mathrm{NMR}$ 谱图中, 4 的谱图变化也较为 明显，随着 $\mathrm{C}-\mathrm{Cl}$ 键的断裂和 $\mathrm{C}-\mathrm{O}$ 键的形成, $\mathrm{CH}_{2}$ 中碳 的吸收峰向低场移动, 从 $\delta 45.61$ 移至 $\delta 64.9$; 另随着原 $\mathrm{C}=\mathrm{O}$ 键变成 $\mathrm{C}=\mathrm{C}-\mathrm{O}$ 键, $\mathrm{C}=\mathrm{C}-\mathrm{O}$ 中 $\mathrm{C}$ 的吸收峰向高 场移动, 从 $\delta 160.7$ 移至 $\delta 144.5$.

\section{3 晶体结构}

化合物 4 的分子结构图见图 3. 晶体结构解析表明, 化合物 4 (CCDC: 975730) 属单斜晶系, $P 2_{1} / n$ 空间群，晶 胞参数 $a=7.4950(15) \AA, b=6.0350(12) \AA, c=22.412(5)$ $\AA, \beta=93.08(3)^{\circ}, V=1012.3(4) \AA^{3}, Z=4, F(000)=480, D_{\mathrm{x}}$ $=1.524 \mathrm{~g} \bullet \mathrm{cm}^{-3}, \mu=0.30 \mathrm{~mm}^{-1}, R\left[F_{2}>2 \sigma\left(F_{2}\right)\right]=0.064$, $w R\left(F_{2}\right)=0.192[I>2 \sigma(I)]$.

如图 3 所示，晶体 4 中的噁二嗪环中的所有原子基 本处在同一平面，噁二嗪环与苯并噻吩环也基本处于平 行状态, 其二面角为 $3.1(5)^{\circ}$. 另外, 在该晶体结构中存 在分子间 $\mathrm{N}-\mathrm{H} \cdots \mathrm{O}$ 氢键和弱的 $\mathrm{S} \cdots \mathrm{S}$ 键, 它们的存在 有利于降低体系能量, 稳定晶体结构, 并进一步形成分 子的空间三维结构. 


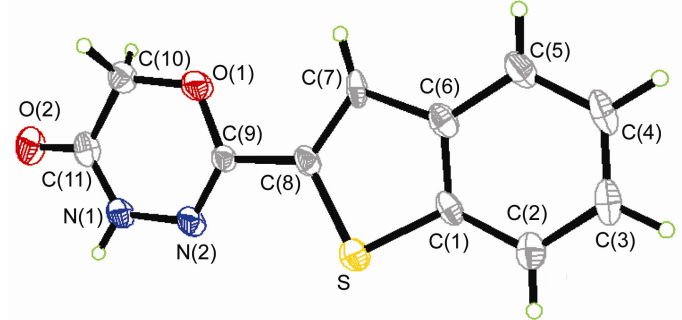

图 3 化合物 $\mathbf{4}$ 的分子结构图

Figure 3 Molecular structure of compound 4

\section{2 结论}

以苯并噻吩-2-甲酸为原料, 设计合成了一种新型 化合物 2-(2-苯并噻吩基)-4H-1,3,4-啞二嗪-5(6H)-酮(4). 该化合物具有潜在的杀虫和除草性能, 有待进一步进行 药效检测. 同时还可设计合成其系列衍生物, 对其研究 还可进一步深入.

\section{3 实验部分}

\section{1 仪器与试剂}

XT4A 显微熔点测定仪(控温型)(北京市科仪电光 仪器厂), 温度未校正; ZF7 三用紫外分析仪(巩义市予华 仪器有限责任公司); BRUKER DRX300 型核磁共振仪 (德国 BRUKER 公司); FIR-360 红外光谱仪(美国 Nicolet 公司); Mircomass Q-TOF micro 型质谱仪; Vario EL III 元素自动分析仪(美国 Elementar 公司); Bruker Smart Apex IICCD 单晶衍射仪. 所用试剂均为国产分析纯, 未 经进一步提纯.

\section{2 实验方法}

\section{2 .1 化合物 1 的合成}

将 $14.25 \mathrm{~g}$ (80 mmol)吲哚-2-甲酸溶于 $500 \mathrm{~mL}$ 甲醇 中, 加入 $2 \mathrm{~mL}$ 浓硫酸, $75{ }^{\circ} \mathrm{C}$ 下回流反应 $24 \mathrm{~h}, \mathrm{TLC}(5 \%$ 水-乙腈)监测反应进程. 反应结束后, 减压蒸除大部分 溶剂, 溶液于 $0{ }^{\circ} \mathrm{C}$ 下冷却静置约 $10 \mathrm{~h}$, 析出针状晶体, 过滤得苯并噻吩-2-甲酸甲酯(1) $15.36 \mathrm{~g}$, 收率 93\%. m.p. $69.3 \sim 69.6{ }^{\circ} \mathrm{C}$ (文献值 ${ }^{[21]}: 69 \sim 71{ }^{\circ} \mathrm{C}$ ); ${ }^{1} \mathrm{H}$ NMR $(300$ $\left.\mathrm{MHz}, \mathrm{CDCl}_{3}\right) \delta: 8.07(\mathrm{~s}, 1 \mathrm{H}), 7.97 \sim 7.75(\mathrm{~m}, 2 \mathrm{H}), 7.53 \sim$ 7.33 (m, 2H), 3.95 (s, 3H); IR (KBr) v: 2949, 2845, 1973, 1938, 1905, 1838, 1719, 1591, 1558, 1521, 1457, 1439, 1336, 1289, 1247, 1174, 1153, 1078, 1060, 924, 871, 761, 723, $696 \mathrm{~cm}^{-1}$; ESI-MS m/z: $215.0[\mathrm{M}+\mathrm{Na}]^{+}$. Anal. calcd for $\mathrm{C}_{10} \mathrm{H}_{8} \mathrm{O}_{2} \mathrm{~S}$ : C 62.48, $\mathrm{H} 4.19$; found $\mathrm{C} 62.41, \mathrm{H} 4.21$.

\section{2 .2 化合物 2 的合成}

将 $7.68 \mathrm{~g}(40 \mathrm{mmol}) 1$ 溶于 $500 \mathrm{~mL}$ 甲醇中, 加入 $23.12 \mathrm{~g}(0.36 \mathrm{~mol})$ 水合肼, $80{ }^{\circ} \mathrm{C}$ 下回流反应 $20 \mathrm{~h}, \mathrm{TLC}$
(5\%水-乙腈)监测反应. 反应结束后, 减压蒸除大部分 溶剂, 溶液于 $0{ }^{\circ} \mathrm{C}$ 下冷却静置约 $10 \mathrm{~h}$, 析出针状晶体, 过滤得苯并塞吩-2-甲酰肼(2) 7.06 g, 收率 92\%. m.p. $184.9 \sim 185.9{ }^{\circ} \mathrm{C}$ (文献值 ${ }^{[22]}$ : $184 \sim 185{ }^{\circ} \mathrm{C}$ ); ${ }^{1} \mathrm{H} \mathrm{NMR}$ $\left(300 \mathrm{MHz}, \mathrm{DMSO}-d_{6}\right) \delta: 10.11(\mathrm{~s}, 1 \mathrm{H}), 8.02(\mathrm{~d}, J=8.5 \mathrm{~Hz}$, 2H), 7.92 (dd, $J=5.8,2.9 \mathrm{~Hz}, 1 \mathrm{H}), 7.44$ (p, $J=7.4 \mathrm{~Hz}$, 2H), $5.10(\mathrm{~s}, 2 \mathrm{H})$; IR (KBr) v: 3302, 3259, 3210, 3057, 3024, 2850, 1933, 1893, 1867, 1650, 1619, 1567, 1457, 1429, 1349, 1324, 1249, 1209, 1159, 1087, 1067, 970, 932, 873, 858, 822, 730, $684 \mathrm{~cm}^{-1}$; ESI-MS m/z: $215.0[\mathrm{M}+$ $\mathrm{Na}]^{+}, 191.0[\mathrm{M}-\mathrm{H}]^{-}$. Anal. calcd for $\mathrm{C}_{9} \mathrm{H}_{8} \mathrm{~N}_{2} \mathrm{OS}$ : C 56.23, H 4.19, N 14.57; found C 56.20, H 4.22, N 14.60.

\section{2 .3 化合物 3 的合成}

将 $4.5 \mathrm{~g}(25 \mathrm{mmol}) 2$ 溶于 $160 \mathrm{~mL} \mathrm{DMF}$ 中, 加入 $2.92 \mathrm{~g}(27.5 \mathrm{mmol})$ 三乙胺, 溶液冷却至 $0{ }^{\circ} \mathrm{C}$, 缓慢滴加 入 $4.90 \mathrm{~g}(37.5 \mathrm{mmol})$ 氯乙酰氯, $0{ }^{\circ} \mathrm{C}$ 下反应 $4 \mathrm{~h}, \mathrm{TLC}$ $(50 \%$ 乙酸乙酯一石油醚)监测反应. 反应结束后, 先过滤 除去白色沉淀物, 减压蒸除约 $2 / 3$ 溶剂, 室温下冷却静 置有固体析出, 过滤得白色固体, 乙酸乙酯重结晶, 得 1-(苯并噻吩-2-甲酰基)-2-(氯乙酰基)肼(3) $5.36 \mathrm{~g}$, 收率 $80 \%$. m.p. $219.5 \sim 221.2{ }^{\circ} \mathrm{C} ;{ }^{1} \mathrm{H}$ NMR $(300 \mathrm{MHz}$, DMSO- $\left.d_{6}\right) \delta: 10.81(\mathrm{~s}, 1 \mathrm{H}), 10.44(\mathrm{~s}, 1 \mathrm{H}), 8.17(\mathrm{~s}, 1 \mathrm{H})$, $8.02(\mathrm{dd}, J=18.8,7.4 \mathrm{~Hz}, 2 \mathrm{H}), 7.58 \sim 7.40(\mathrm{~m}, 2 \mathrm{H}), 4.22$ $(\mathrm{s}, 2 \mathrm{H}) ;{ }^{13} \mathrm{C}$ NMR $\left(75 \mathrm{MHz}, \mathrm{DMSO}-d_{6}\right) \delta: 165.5,160.7$, $140.2,138.9,136.9,126.5,126.0,125.4,125.0,122.8$, 45.4; IR (KBr) v: 3424, 3264, 3068, 2937, 2800, 2738, 2677, 1943, 1910, 1691, 1648, 1564, 1550, 1521, 1500, 1428, 1404, 1343, 1303, 1231, 1204, 1140, 1069, 1036, 977, 942, 884, 841, 789, 757, 720, $637 \mathrm{~cm}^{-1}$; ESI-MS m/z: $291.0[\mathrm{M}+\mathrm{Na}]^{+}, 267.0[\mathrm{M}-\mathrm{H}]^{-}$. Anal. calcd for $\mathrm{C}_{11} \mathrm{H}_{9} \mathrm{ClN}_{2} \mathrm{O}_{2} \mathrm{~S}$ : C 49.17, $\mathrm{H} 3.38, \mathrm{~N} \mathrm{10.42}$; found $\mathrm{C} 49.13$, H 3.40, N 10.4.

\section{2 .4 化合物 4 的合成}

将 $2.69 \mathrm{~g}$ (10 mmol) 3 加热溶于 $50 \mathrm{~mL} \mathrm{DMF}$ 中, 加 入 $6.9 \mathrm{~g} \mathrm{(50} \mathrm{mmol)}$ 碳酸钾, $80{ }^{\circ} \mathrm{C}$ 下反应 $6 \mathrm{~h}$, TLC (50\%乙 酸乙酯一石油醚)监测反应, 反应结束后, 先过滤除去沉 淀物, 滤液中加入 $50 \mathrm{~mL}$ 水, 溶液立即析出固体成悬浊 液, 冷却静置一夜, 过滤得淡黄色固体 2-(2-苯并噻吩 基)-4H-1,3,4-噁二嗪-5(6H)-酮(4) $1.11 \mathrm{~g}$, 收率 48\%. m.p. $237.9 \sim 238.9{ }^{\circ} \mathrm{C} ;{ }^{1} \mathrm{H}$ NMR (300 MHz, DMSO- $\left.d_{6}\right) \delta$ : 11.12 (s, 1H), 7.94 (dd, $J=20.6,8.5 \mathrm{~Hz}, 2 \mathrm{H}), 7.79$ (s, 1H), 7.42 (p, $J=7.1 \mathrm{~Hz}, 2 \mathrm{H}), 4.83$ (s, 2H); ${ }^{13} \mathrm{C}$ NMR (75 MHz, DMSO- $\left.d_{6}\right) \delta: 160.8,144.5,139.7,138.9,132.9,126.0$, 125.0, 124.8, 124.7, 122.4, 64.9; IR (KBr) v: 3347, 3242, 3125, 3097, 3067, 2898, 1963, 1923, 1885, 1748, 1686, 
$1634,1560,1529,1457,1398,1328,1306,1247,1182$, 1161, 1144, 1057, 1015, 959, 938, 864, 809, 758, 727, 632 $\mathrm{cm}^{-1}$; ESI-MS m/z: $255.0[\mathrm{M}+\mathrm{Na}]^{+}, 231.0[\mathrm{M}-\mathrm{H}]^{-}$. Anal. calcd for $\mathrm{C}_{11} \mathrm{H}_{8} \mathrm{~N}_{2} \mathrm{O}_{2} \mathrm{~S}$ : C 56.88, H 3.47, $\mathrm{N}$ 12.06; found C 56.84, H 3.50, N 12.10.

\section{3 晶体结构测定}

取 $10 \mathrm{mg}$ 化合物 4 置于 $25 \mathrm{~mL}$ 烧杯中, 用乙酸乙酯 和正已烷加热溶解, 薄膜封口后留小孔, 待溶剂缓慢挥 发后, 析出适宜于单晶结构解析的晶体.

将化合物 4 的单晶 $(0.30 \mathrm{~mm} \times 0.20 \mathrm{~mm} \times 0.10 \mathrm{~mm})$ 置于 $X$ 衍射仪上, 于 $293 \mathrm{~K}$ 用经石墨单色器单色化的 Mo K $\alpha$ 射线 $(\lambda=0.71073 \AA)$, 以 $\omega / 2 \theta$ 扫描方式收集衍射 数据 1999 个, 其中 $I>2 \sigma(I)$ 独立衍射点为 1167 个, 用于 结构分析. 晶体结构由直接法解出, 以 XSCANS 程序寻 找衍射峰后精确测定出晶胞参数. 所有计算结果由 SHELXL-97 程序完成, 全部非氢原子坐标和各向异性 温度因子经全矩阵最小二乘法修正.

\section{辅助材料(Supporting Information) 所成产物 1 4 的} ${ }^{1} \mathrm{H}$ NMR 谱图、FT-IR 谱图、ESI-MS 谱图和产物 3, 4 的 ${ }^{13} \mathrm{C} \mathrm{NMR}$ 谱图. 这些材料可以免费从本刊网站 (http://sioc-journal.cn/)上下载.

\section{References}

[1] Sieardi, S. M.; Landam, S.; Gaozza, C. H. J. Heterocycl. Chem. 1973, 10, 1039.

[2] Li, Y.-X. Hunan Chem. Ind. 1995, 25, 12 (in Chinese). (李玉新，湖南化工, 1995, 25, 12.)

[3] Stephen, F. M.; Gary, D.; Annis, R. S.; David, W. P. Pest Manage. Sci. 2001, 57, 153.

[4] Cao, S.; Qian, X.-H.; Song, G.-H. J. Fluorine Chem. 2002, 117, 63.

[5] Liu, C.-L. Pesticides 2004, 24, 42 (in Chinese).
(刘长令, 农药, 2004, 24, 42.)

[6] Gaonkar, S. L.; Rai, K. M. L.; Prabhuswamy, B. Eur. J. Med. Chem. 2006, 41, 841 .

[7] Jeffrey, L. R.; Scott, W. M.; Nicholas, A. M. J. Med. Chem. 2007, $50,528$.

[8] Ke, S.-Y.; Qian, X.-H.; Li, Z. Eur. J. Med. Chem. 2009, 44, 2113.

[9] Dong, M.; Cao, J.; Chen, L.; Lü, D.-H.; Hu, J.; Shen, Z.; Ni, C.-C.; Zhang, Y.-B.; Yuan, L.-P. Agrochemicals 2009, 48, 726 (in Chinese).

(董敏, 曹瑾, 陈亮, 吕冬华, 胡军, 沈宙, 倪长春, 张一宾, 袁 莉萍, 农药, 2009, 48, 726.)

[10] Sun, L.; Cao, J.; Chen, L.; Lü, D.-H.; Ni, C.-C.; Shen, Z.; Yuan, L.-P.; Zhang, Y.-B. Chin. J. Pestic. Sci. 2010, 12, 221 (in Chinese). (孙亮, 曹瑾, 陈亮, 吕冬华, 倪长春, 沈宙, 袁莉萍, 张一宾, 农药学学报, 2010, 12, 221.)

[11] Pan, L.-L.; Shen, X.-X.; Cao, J.; Chen, L.; Shen, Z.; Yuan, L.-P.; Zhang, Y.-B. Chin. J. Pestic. Sci. 2011, 13, 239 (in Chinese). (潘玲玲, 沈晓霞, 曹瑾, 陈亮, 沈宙, 袁莉萍, 张一宾, 农药学 学报, 2011, 13, 239.)

[12] Walter, H. WO 0034286, 2000 [Chem. Abstr. 2000, 133, 30738d].

[13] Demirayak, S.; Mohsen, U. A. Heterocycl. Chem. 2001, 38, 507.

[14] Jennings, L. D.; Kincaid, S. L.; Wang, Y. D.; Krishnamurthy, G.; Beyer, C. F.; McGinnis, J. P.; Miranda, M.; Discafani, C. M.; Rabindran, S. K. Bioorg. Med. Chem. Lett. 2005, 15, 4731.

[15] Wang, H.-M.; Guo, S.-B.; Hu, Z.-G.; Zeng, X.-H.; Yang, G.-Y. Chin. J. Org. Chem. 2015, 35, 1075 (in Chinese).

(王红梅, 郭树兵, 胡扬根, 曾小华, 杨光义, 有机化学, 2015, 35, 1075.)

[16] Wu, Q.; Wu, Q.; Zhang, X.; Yang, J.-C.; Liu, C.-L. Agrochemicals 2010, 49, 5 (in Chinese).

(吴崕, 伍强, 张茜, 杨吉春, 刘长令, 农药, 2010, 49, 5.)

[17] Tietze, L. F.; Lohmann, J. K.; Stadler, C. Synlett 2004, 1113.

[18] Leonczak, P.; Gao, L.-J.; Ramadori, A. T.; Lescrinier, E.; Rozenski, J.; Jonghe, S. D.; Herdewijn, P. ChemMedChem 2014, 9, 2587.

[19] Shiota, T.; Yamagami, S.; Kataoka, K.; Endo, N.; Barnum, D.; Greene, J.; Moree, W.; Weinhouse, M. R.; Tarby, C. M. US 6686353, 2004 [Chem. Abstr. 2004, 140, 163894].

[20] Alphen, J. V. Recl. Trav. Chim. Pays-Bas 1929, 48, 417.

[21] Ahn, S. H.; Jang, S. S.; Kim, Y. K.; Lee, K. J. Bull. Korean Chem. Soc. 2012, 33, 233.

[22] Weissgerber; Kruber Chem. Ber. 1920, 53, 1564. 